\title{
Acerca de la influencia de la teoría del Estado de Johann G. Fichte en la ética de Hermann Cohen
}

\author{
HÉCTOR OSCAR ARRESE IGOR \\ Universidad Nacional de La Plata \\ Facultad de Humanidades y Ciencias de la Educación \\ Departamento de Filosofía \\ harreseigor@gmail.com
}

\begin{abstract}
Resumen: En este trabajo intento mostrar que existen diferencias importantes entre las concepciones del Estado de Hermann Cohen y de Johann Gottlieb Fichte. Por lo tanto, concluyo que la tesis de la influencia de la filosofía fichteana en la teoría de Cohen es problemática.

Palabras clave: derecho, democracia, constitución, libertad
\end{abstract}

\begin{abstract}
In this paper I try to show that there are important differences between Hermann Cohen's and Johann Gottlieb Fichte's conceptions of the state. Therefore I conclude that the thesis of the influence of the Fichtean philosophy upon Cohen's theory is problematic.

Key words: right, democracy, constitution, freedom
\end{abstract}

En las últimas décadas hemos asistido a un verdadero renacimiento de los estudios sobre el neokantismo de Marburgo, en especial a partir de la edición de las obras completas de Hermann Cohen a cargo de Helmut Holzhey (Universidad de Zurich). Entre los estudios más importantes sobre la obra de Cohen merecen especial mención las obras de Helmut Holzhey, Andrea Poma, Pierfrancesco Fiorato, Jürgen Stolzenberg, Hartwig Wiedebach y Peter Schmid. Pero la obra que abrió el camino para las nuevas investigaciones en este campo fue la tesis doctoral de Dieter Adelmann ("Einheit des Bewusstseins als Grundproblem der Philosophie Hermann Cohens").

Stolzenberg y Schmid se hicieron eco de las afirmaciones de Heidegger y Gadamer acerca de la fuerte impronta fichteana del neokantismo. ${ }^{1}$ En esta línea, señalaron algunos elementos de la teoría expuesta por Fichte en el Grundlage des Naturrechts que estarían presentes en la Ethik des reinen Willens, sobre todo en relación con el concepto de

1 "Der Neukantianismus war viel eher eine Rückwendung zu Fichte gewesen" (H.G. Gadamer, "Subjektivität und Intersubjektivität, Subjekt und Person", p. 88). Antes de Gadamer, Heidegger ya había afirmado que el neokantismo es más bien un neofichteísmo (M. Heidegger, "Phänomenologie und transzendentale Wertphilosophie. Vorlesung Sommersemester 1919”, pp. 142 ss.).

Diánoia, volumen LVI, número 66 (Mayo 2011): pp. 141-164. 
autoconciencia. Schmid se limitó a sugerir que en ambos casos el yo es pensado como un ser social, pero no avanzó más en esta dirección. ${ }^{2}$ En cambio, Stolzenberg comparó la deducción de la autoconciencia en las dos teorías, mostrando acertadamente que tanto en Fichte como en Cohen la alteridad desempeña un papel fundamental. ${ }^{3}$ Retomando los aportes valiosos de Schmid y Stolzenberg, considero que estas teorías tienen elementos conceptuales en común que hacen plausible la hipótesis de que la filosofía fichteana del derecho habría ejercido alguna influencia sobre la ética de Cohen. Sin embargo, puede objetarse esta afirmación a partir de la constatación de que el concepto de autoconciencia que defiende cada uno es diferente. En la Ethik des reinen Willens se trata de una forma de subjetividad que está en la base del funcionamiento del Estado como persona jurídica; es decir, que la autoconciencia es el sujeto de la voluntad pura que se expresa en el Estado por medio de la actividad legislativa. Por el contrario, Fichte busca las condiciones de posibilidad de la autoconciencia individual, que consiste en una relación que el yo establece consigo mismo.

Sin embargo, el concepto de autoconciencia se deduce en ambas teorías a partir de la alteridad. Cohen explica las relaciones legales a partir de una relación intersubjetiva de tipo contractual, que tiene como resultado la declaración de una voluntad consensuada entre los sujetos. Es decir, Cohen no puede explicar la autoconciencia a partir del yo toma-

2 "[Damit] nimmt Cohen eine fichtesche Position ein. Er verwirft zwar die erkenntnistheoretische Konzeption der Wissenschaftslehre Fichtes, gesteht diesem aber zu, dass er in praktischer Hinsicht durchaus bedenkenswerte Überlegungen angestellt habe. Dies etwa dort, wo er das Ich als soziales Ich entdeckt, als ein Ich, das sich nur in Gemeinschaft mit einem Du konstituieren kann. Diese Überlegungen finden sich vor allem in der 1796 erschienenen 'Grundlage des Naturrechts nach Prinzipien der Wissenschaftslehre', welche zusammen mit der 1794 erschienenen 'Grundlage der gesamten Wissenschaftlehre' das Ziel verfolgt, die freie Selbstbestimmung und damit das Problem der Freiheit zu klären" [Cohen toma una posición fichteana. Él rechaza por cierto la concepción gnoseológica de la teoría de la ciencia de Fichte; sin embargo, concede que Fichte ha realizado consideraciones absolutamente valiosas desde el punto de vista práctico: descubre al yo como un yo social que sólo puede constituirse en comunidad con un tú. Estas reflexiones se encuentran principalmente en el "Fundamento del derecho natural de acuerdo con los principios de la teoría de la ciencia" de 1796, que, junto con el "Fundamento de la totalidad de la teoría de la ciencia" de 1794, persigue el objetivo de aclarar la libre autodeterminación y la libertad] (P. Schmid, Ethik als Hermeneutik. Systematische Untersuchungen zu Hermann Cohens Rechts und Tugendlehre, pp. 60-61).

${ }^{3}$ J. Stolzenberg, "Fichte im Neukantianismus. Probleme der Fichte-Rezeption bei Wilhelm Windelband, Heinrich Rickert, Hermann Cohen und Paul Natorp", pp. 428-432.

Diánoia, vol. LVI, no. 66 (Mayo 2011). 
do aisladamente de los demás, porque el otro también es una condición de posibilidad de la acción legal. Lo mismo ocurre con la fundamentación fichteana de la autoconciencia, dado que parte de la relación en la que el otro exhorta al yo a autodeterminarse a la acción. Esta acción es posible, a su vez, sólo si el yo y el otro entran en una relación de reconocimiento recíproco. Dicho de otro modo, el yo puede devenir autoconsciente sólo si es tratado por el otro como un ser racional y libre, y si le responde a su vez de la misma manera.

También la idea del Estado es un concepto central de ambas teorías y está íntimamente unida a los conceptos de autoconciencia y relación legal, si bien el vínculo es diferente en cada caso. En la Ethik des reinen Willens, el Estado es la mediación de la autoconciencia, que se realiza gracias a su actividad como persona jurídica. En la medida en que los ciudadanos deliberen, acuerden y luego declaren su voluntad común en el Parlamento, el sujeto universal de la autoconciencia adquirirá realidad histórica. De este modo, según Cohen, cada individuo entrará en contacto con la universalidad de la ley.

Fichte otorga un papel diferente a la idea del Estado, porque es el garante de la libertad y la propiedad de los ciudadanos. El Estado es aquí la última condición de la vigencia del principio del derecho, porque el respeto de las esferas de acción de los sujetos no es posible si no se protege su propiedad, entendida como el conjunto de las condiciones de la acción. Pero la autoconciencia sólo se logra gracias a la instauración de la relación legal. Por lo tanto, el Estado opera como una condición de la constitución de la subjetividad.

En este trabajo intentaré mostrar que, más allá de las semejanzas señaladas, existen diferencias profundas entre los dos modelos de Estado, porque Cohen propone una democracia deliberativa, mientras que Fichte aboga por una versión débil de la soberanía popular; por otro lado, la relación entre la moralidad, el derecho y el Estado no es la misma en los dos casos. Para lograr este objetivo, compararé la teoría de Cohen no sólo con el Grundlage des Naturrechts, sino que también tomaré en cuenta los escritos revolucionarios de Fichte, así como los correspondientes al periodo berlinés. Finalmente, indagaré las razones de estos desacuerdos entre las dos teorías, con el objetivo de brindar un aporte al debate sobre la presencia de la filosofía política de Fichte en la Ethik des reinen Willens, una obra fundamental del neokantismo de Marburgo. 


\section{La deducción del Estado como condición de la autoconciencia}

Cohen ha propuesto la idea de un Estado socialista, fundado sobre la actividad parlamentaria y el sufragio universal. Las fuertes críticas de Cohen al capitalismo, en particular con ocasión de sus investigaciones sobre la persona jurídica de la cooperativa, adquieren sentido en el horizonte de un socialismo ético, donde cada persona se autodetermina por medio de la autolegislación. Es famosa su afirmación:

El carácter de fin en sí mismo produce y determina el concepto de persona, el concepto fundamental de la ética. La mercancía es un mero medio en cuanto cosa del tráfico comercial. El trabajador no puede nunca ser confundido con una mera mercancía, aunque sea para los fines supremos de la riqueza nacional; él debe ser considerado y tratado "en todo momento y al mismo tiempo como fin". 4

Cohen ve en la ética kantiana la fuente de inspiración del ideal socialista, lo que convierte a Kant, en su opinión, en el verdadero fundador del socialismo alemán. ${ }^{5}$ Como es sabido, la idea de un socialismo kantiano desempeñó un papel fundamental en el debate revisionista en el interior del Partido Socialdemócrata Alemán (SPD), en la Alemania de fines del siglo XIX y comienzos del XX.

Cohen se ocupa de la teoría del Estado para demostrar que la autoconciencia es la categoría central de la ciencia del derecho. El sistema legal es idéntico al Estado mismo, porque el Estado es una persona jurídica y, por lo tanto, su existencia depende de la expresión de la voluntad común de los ciudadanos por medio de la legislación. ${ }^{6}$ Dado que Cohen identifica al Estado con la totalidad del sistema del derecho, y con las condiciones de su producción y aplicación, es indispensable demostrar, en primer lugar, que el Estado no puede ser pensado sin el

${ }^{4}$ H. Cohen, Einleitung mit kritischem Nachtrag, p. 113: "Der Selbstzweck erzeugt und bestimmt den Begriff der Person, den Grundbegriff der Ethik. Bloßes Mittel ist die Sache, die als Sache des wirtschaftlichen Verkehrs die Ware ist. Der Arbeiter kann daher niemals bloß als Ware zu verrechnen sein, auch für die höheren Zwecke des angeblichen Nationalreichtums nicht; er muß 'jederzeit zugleich als Zweck' betrachtet und behandelt werden." Cfr. H.L. Ollig, Der Neukantianismus, p. 127.

${ }^{5}$ H. Cohen, Einleitung mit kritischem Nachtrag, p. 112. Cfr. el comentario de T. Meyer en "Eine unzeitgemäße Intervention, die an der Zeit war. Hermann Cohens neukantianischer Sozialismus und die sozialdemokratische Ideologie im Kaiserreich", p. 261.

${ }^{6}$ Cfr. H. Holzhey, "Hermann Cohen: der Philosoph in Auseinandersetzung mit dem politischen und gesellschaftlichen Probleme seiner Zeit", pp. 27-28.

Diánoia, vol. LVI, no. 66 (Mayo 2011). 
concepto de autoconciencia, para luego poder concluir que el derecho no puede tampoco ser explicado sin esta idea.

Cohen recurre a sus investigaciones sobre la persona jurídica de la cooperativa para determinar el tipo específico de persona jurídica que es el Estado. Como resultado de sus reflexiones sobre el estatus legal de la cooperativa, Cohen concluye en el capítulo 4 de la Ethik des reinen Willens que la voluntad común resultante del acuerdo de los cooperadores es independiente de sus voluntades fácticas, porque adquiere autonomía respecto de los cambios futuros en las preferencias y el parecer de los individuos que se han sometido a ella. ${ }^{7}$ Dicho de otro modo, los cooperadores ya no pueden decidir cuáles normas obedecerán y cuáles no, sino que deberán someterse de allí en adelante a las normas que ellos mismos han debatido y luego acordado. Lo mismo ocurre en la persona jurídica del Estado, puesto que los ciudadanos no pueden cambiar caprichosamente y a discreción las normas que no les convengan.

Ahora bien, la voluntad común del Estado se expresa cada vez que el poder legislativo proclama una nueva ley. ${ }^{8}$ Se trata de una voluntad declarada y expresa, pero que a su vez es el producto de la deliberación y la discusión previas. Es decir, se trata de una voluntad que tiene una unidad, porque se la ha moldeado a lo largo del debate y presenta una coherencia interna. En algunos puntos, seguramente esta voluntad que se ha proclamado discrepará al menos en parte del contenido que muchas de las voluntades particulares tenían antes de comenzar la tarea de la legislación, porque la homogeneidad de la voluntad común es el resultado de la deliberación y la negociación.

La voluntad común que se expresa en cada ley es diferente en cada caso, de acuerdo con el contenido del que se trate; sin embargo, la voluntad que se expresa en cada acto de legislación es la expresión de una misma voluntad común, que no es otra que la voluntad del pueblo que se expresa en la Constitución. El contenido de la voluntad constitucional consiste en las reglas del procedimiento que debe seguirse en la legislación para garantizar que ésta sea efectivamente democrática. Se trata de condiciones tales como la exigencia del sufragio universal para elegir a los legisladores, la igualdad de voz y voto en el Parlamento, la ausencia de coacción sobre los representantes, su eventual inmunidad parlamentaria, etcétera. ${ }^{9}$

${ }^{7}$ H. Cohen, Ethik des reinen Willens, p. 243.

${ }^{8}$ H.L. Ollig, Religion und Freiheitsglaube, p. 156; K. Lisser, Der Begriff des Rechts bei Kant. Mit einem Anhang über Cohen und Görland, p. 55.

${ }^{9}$ La impugnación de Cohen del sistema prusiano de votación por clases está motivada también por el reclamo de inclusión de la minoría judía en la vida es- 
Una vez que la voluntad común se ha expresado y fijado por escrito en la Constitución, entonces bastará simplemente con que se controle el respeto por las normas allí establecidas para que la voluntad estatal sea efectivamente una voluntad común y universal. La vigencia de la ley fundamental garantiza la universalidad de las normas promulgadas, sin necesidad de que todos los ciudadanos participen a cada momento en la tarea de la legislación. La personalidad jurídica del Estado consiste, entonces, en la expresión de la voluntad común, o sea en la construcción de cierto tipo de subjetividad que, al igual que en el caso de la persona jurídica de la cooperativa, es el sujeto de una voluntad ficticia y técnico-legal.

Dicho de otro modo, la persona jurídica del Estado es la expresión de una voluntad pura, que no depende de la voluntad fáctica y actual de cada ciudadano, ni tampoco de un grupo de ellos. ${ }^{10}$ Se trata del sujeto de una voluntad que surge a partir de sí misma, sin otra referencia que las reglas que la constituyen. El Estado es, entonces, un sujeto o una persona universal en virtud de su voluntad. El Estado constituye la autoconciencia o, lo que es lo mismo, es el sujeto de la ética. ${ }^{11}$ Toda vez que el Estado es la expresión de la voluntad pura, y el contenido del movimiento de la voluntad pura es la tarea (Aufgabe), entonces el

tatal (P. Schmid, Ethik als Hermeneutik. Systematische Untersuchungen zu Hermann Cohens Rechts und Tugendlehre, p. 77). Cfr. H. Holzhey, "Hermann Cohen: der Philosoph in Auseinandersetzung mit dem politischen und gesellschaftlichen Probleme seiner Zeit", pp. 27-28; E. Winter, Ethik und Rechtswissenschaft. Eine historischsystematische Untersuchung zur Ethik-Konzeption des Marburger Neukantianismus im Werke Hermann Cohens, pp. 333-334. Acerca de la superación de las pretensiones hegemónicas de la religión en la universalidad del Estado, cfr. H. Lübbe, "Die politische Theorie des Neukantianismus und des Marxismus", p. 234; "Neukantianische Sozialismus", p. 240. Para la cuestión de la neutralidad estatal, cfr. S. Schwarzschild, "The Democratic Socialism of Hermann Cohen", p. 216.

${ }^{10}$ P. Giesecke, "Kant und der Sozialismus. Studien zum Marburger Neukantianismus, Philosophisches Kritizismus und Kritischen Rationalismus", p. 119; C. Müller, Die Rechtsphilosophie des Marburger Neukantianismus. Naturrecht und Rechtpositivismus in der Auseinandersetzung zwischen Hermann Cohen, Rudolf Stammler und Paul Natorp, p. 129; P. Natorp, "Hermann Cohens philosophische Leistung unter dem Gesichtspunkte des Systems", p. 97.

${ }^{11} \mathrm{H}$. Cohen, Ethik des reinen Willens, p. 80. Acerca del Estado como concepto modelo de la autoconciencia, cfr. P. Schmid, Ethik als Hermeneutik. Systematische Untersuchungen zu Hermann Cohens Rechts und Tugendlehre, pp. 69-70; E. Winter, Ethik und Rechtswissenschaft. Eine historisch-systematische Untersuchung zur EthikKonzeption des Marburger Neukantianismus im Werke Hermann Cohens, p. 349; A. Görland, "Hermann Cohens systematische Arbeit im Dienste des kritischen Idealismus", p. 240.

Diánoia, vol. LVI, no. 66 (Mayo 2011). 
Estado mismo es la tarea de constituir el sujeto de la voluntad pura. Por lo tanto, el Estado es la tarea de la autoconciencia. ${ }^{12}$ El Estado significa, entonces, la unidad del objeto y el sujeto de la voluntad, porque el objeto de la voluntad es la expresión de sí misma, y con ella del sujeto de esta voluntad. ${ }^{13}$

Por otro lado, Cohen sostiene que las voluntades individuales y fácticas de los ciudadanos pueden ordenarse para la realización de la tarea de la autoconciencia. Cada ciudadano se orienta a la constitución de la autoconciencia en la voluntad común del Estado, en virtud de la actividad parlamentaria de sus representantes. Por medio del sufragio, cada ciudadano delega en los legisladores la representación de su voluntad. Si no estuviera garantizado el sufragio universal, entonces la voluntad común sería una mera farsa, porque no unificaría ni integraría las voluntades individuales. ${ }^{14}$

Otra cuestión importante que plantea la realización de la autoconciencia en el Estado es el tipo de subjetividad del que aquí se trata. Dado que la autoconciencia es el sujeto de la ética, puede ser pensada en analogía con el concepto del objeto de la naturaleza, del que se ocupa la lógica. Sin embargo, no se puede llevar más lejos la analogía con la ciencia natural afirmando que la autoconciencia está constituida por las voluntades individuales de los ciudadanos, que están incluidas en ella, del mismo modo que los órganos lo están en el organismo. El problema radica en que el sujeto no puede ser dividido en partes, porque es espíritu expresado en la organización legal del Estado.

${ }^{12}$ H. Cohen, Ethik des reinen Willens, p. 244. M. Dreyer, Die Idee Gottes im Werk Hermann Cohens, p. 123.

${ }^{13}$ H. Cohen, Ethik des reinen Willens, p. 245.

14 "Porque, como la universalidad del Estado, en cuanto unidad y tarea de la unidad, está condicionada por la voluntad particular, el particular debe tomar en sí esta tarea de la universalidad; debe ordenarse a esta unidad e integrarse en ella. Esta unidad es el contenido, con el que debe relacionarse su voluntad en todas sus acciones. Y la voluntad particular puede conseguir y afirmar su sujeto, su autoconciencia, como tarea de su voluntad pura, sólo en este contenido, en este objeto. Contra esto se erizan sobre todo los impulsos del absolutismo religioso y la soberanía sobre todas las tendencias del espíritu humano" ["Denn wie die Allheit des Staates, als Einheit, und zwar als Aufgabe der Einheit, durch den Einzelwillen bedingt ist, so hat der Einzelne diese Aufgabe der Allheit auf sich selbst zu nehmen; dieser Einheit sich einzuordnen und einzugliedern. Diese Einheit ist der Inhalt, auf den er in allen seinen Handlungen seinen Willen zu beziehen hat, Und nur in diesem Inhalt, in diesem Objekte kann er sein Subjekt, sein Selbstbewusstsein, als die Aufgabe seines reinen Willens, erlangen und behaupten. Dagegen sträuben sich hauptsächlich die Regungen der religiösen Absolutheit und Souveränität für alle Richtungen des Menschengeistes"] (H. Cohen, Ethik des reinen Willens, p. 245). 
No se trata del espíritu que se hace presente en la comunidad religiosa, entendido como una emanación de la divinidad. Ni tampoco del espíritu en el sentido de una formación de la conciencia cultural totalmente independiente del conocimiento científico, al modo del espíritu que se expresa en el arte. Se trata, en realidad, del espíritu propio de la conciencia cultural que se manifiesta en la ciencia del derecho.

El espíritu surge cuando los ciudadanos hallan la formulación escrita de una voluntad común a la que han llegado; por lo tanto, supone siempre el acuerdo previo entre ellos. En este sentido, el contrato es el fundamento de la persona jurídica del Estado y, dado que el Estado es idéntico a la totalidad del sistema legal, es el fundamento del derecho mismo. Toda relación legal puede ser pensada bajo la forma del contrato, porque en realidad la legislación es un acto de deliberación y acuerdo entre adultos consintientes en condiciones de simetría. Dicho de otro modo, "la autoconciencia se realiza en el contrato y está condicionada por él". ${ }^{15}$

La autoconciencia hace posible una comunidad espiritual entre los ciudadanos, porque la forma contractual que adquiere la legislación en el Estado liga a los ciudadanos con los lazos del mutuo reconocimiento como sujetos de derecho. El otro deja de ser un extraño porque participa en la empresa común de la legislación y en la formación de la voluntad estatal por medio de la unificación de las voluntades particulares del otro y el yo. Los ciudadanos construyen la comunidad espiritual del Estado - la autoconciencia- en la actividad legislativa.

Cohen ejemplifica la relación intersubjetiva que la autoconciencia instaura entre los ciudadanos con el carácter de reclamabilidad judicial de la acción legal. Veamos de qué manera lo hace. La relación legal es una actio, en el sentido de que contiene en sí no sólo las normas que deben cumplirse, sino también el procedimiento que ha de seguirse para denunciar y procesar a quien las transgreda. Una acusación penal (Anklage) sólo puede llevarse a cabo si se determinan con claridad al menos dos personas físicas: la del querellante y la del acusado. Es decir, que se trata de una relación entre un yo y un tú concretos y específicos. Como dice Cohen:

Pero allí yace al mismo tiempo la exigencia [...] de que yo no pueda pensar en "Yo" sin pensar en ti. Así, el otro se ha transformado en la autoconciencia en cierto modo en el Dualis del yo. En la medida en que la autoconciencia debe significar la unidad de la voluntad, debe formar la

${ }^{15}$ H. Cohen, Ethik des reinen Willens, p. 248: "Das Selbstbewusstsein [...] vollzieht sich in dem Vertrage und ist durch ihn bedingt." 
unión de tú y yo. La voluntad me une a mí contigo; a ti conmigo. Esta unidad significa la tarea de la autoconciencia. ${ }^{16}$

Cohen considera que esta característica intrínseca de la ley —que establece una correlación necesaria entre por lo menos dos sujetos-constituye un progreso en la relación intersubjetiva, que va desde una primera relación con un otro general y abstracto, hasta la correlación con un tú siempre concreto, sea en calidad de querellante o de acusado. Si el derecho no pudiera ser pensado bajo la forma del contrato, no estaría constituido en la forma de un sujeto colectivo, esto es, de la persona jurídica del Estado. Pero entonces la autoconciencia tampoco sería la categoría fundamental del Estado y la ética no podría deducirse a partir del derecho estatal. La autoconciencia, entonces, supera la posición del yo aislado y separado de los demás, y lo lleva a convertirse en un tú. Dicho de otro modo, la autoconciencia instituye una comunidad tan íntima entre el yo y el tú que no puede pensarse el uno sin el otro.

En la Ethik des reinen Willens se trata de la conciencia de esa forma de subjetividad que Cohen denomina como el "sí mismo" (Selbst), para diferenciarla de la autoconciencia del yo. ${ }^{17}$ Cohen sospecha que el concepto de autoconciencia individual está teñido de psicologismo; es decir, que la autoconciencia del individuo no es más que la conciencia empírica de sus estados internos. Pero no puede fundamentarse ninguna ciencia a partir de la supuesta evidencia de estados internos de conciencia a los que sólo el sujeto puede tener acceso. El yo sólo es una parte de la voluntad pura que unifica el querer del yo y del tú. Si el yo y el tú no son pensados juntos y correlativamente, la idea misma de voluntad pura carece de sentido. Como dice el propio Cohen:

El sí mismo no es algo así como la fuerza natural dada psicológicamente, que es quien produce la voluntad; y en este caso sería pensada como un poder natural y una disposición garantizados; pero más bien es siempre sólo la tarea quien constituye, en cuanto tal, tanto al sujeto cuanto al objeto del querer puro. ${ }^{18}$

${ }^{16}$ H. Cohen, Ethik des reinen Willens, p. 249: "Aber es liegt darin zugleich die [...] Forderung: dass ich auch nicht Ich denken kann, ohne dich zu denken. So hat der Andere im Selbstbewusstsein sich gleichsam in den Dualis des Ich verwandelt. Wenn das Selbstbewusstsein die Einheit des Willens zu bedeuten hat, so muss sie die Vereinigung von Ich und Du bilden. Der Wille vereinigt mich und dich; dich und mich. Diese Einheit bedeutet die Aufgabe des Selbstbewusstseins."

${ }^{17} \mathrm{H}$. Cohen, Ethik des reinen Willens, p. 258.

${ }^{18}$ Ibid., p. 259: "das Selbst ist nicht etwa die psychologisch gegebene Naturkraft, 
Cohen estructura el concepto de la autoconciencia con base en la tarea, en tanto que es el motivo de la voluntad pura; pero la voluntad pura se expresa como autoconciencia en la voluntad estatal, que dota al Estado de personalidad jurídica. Por lo tanto, la autoconciencia es la realización de la voluntad pura en la acción legal, que incluye tanto la tarea de la legislación cuanto la aplicación de la ley. Esto es,

El concepto de ley, al que aquí se está haciendo referencia, no tiene que ver con la unidad y la esencia de la leyes, que son establecidas por la ley fundante, la Constitución; sino más bien con las leyes particulares, en las cuales se atestiguan y realizan las tareas de la voluntad estatal. Sin ley no hay voluntad, y por lo tanto tampoco una autoconciencia del Estado. Este concepto estatal de la ley debe convertirse en el concepto guía para la autoconciencia personal. ${ }^{19}$

La tarea de la autoconciencia en la voluntad estatal es el objetivo último de la moralidad que, a su vez, es el norte de la vida política. Nunca puede decirse que se ha completado la tarea de la autoconciencia, porque es infinita y está siempre abierta al futuro.

\section{La idea del Estado a lo largo de la obra de Fichte}

Fichte se interesó por cuestiones de teoría política ya desde sus primeros escritos. En sus famosos textos sobre la Revolución Francesa (Zurückforderung der Denkfreiheit, de 1793, y Beitrag zur Berichtigung der Urtheile des Publicums über die französische Revolution, de 1794), Fichte parte del principio de que "el hombre no puede ser heredado, vendido o regalado" ("Der Mensch kann weder ererbt, nocht verkauft, noch verschenkt werden"). ${ }^{20}$ Por esta razón el ciudadano debe obedecer solamente aquellas normas que él se ha dado a sí mismo. De este

welche den Willen hervorbringt; und wäre sie noch so primitiv als eine verborgene Naturmacht und Anlage gedacht; sondern es ist immer nur die Aufgabe, welche, als solche, so das Subjekt, wie das Objekt des reinen Wollens bildet".

${ }^{19} \mathrm{H}$. Cohen, Ethik des reinen Willens, p. 262: "Bei dem Gesetze, wie es hier gemeint ist, handelt es sich aber nicht um die Einheit und den Inbegriff der Gesetze, den das Grundgesetz, die Verfassung ausmacht; sondern um die einzelnen Gesetze selber, in denen die Aufgaben des Staatswillens sich bekunden und betätigen. Ohne Gesetz kein Wille, also auch kein Selbstbewusstsein des Staates. Dieser Staatsbegriff des Gesetzes muss der Leitbegriff werden für das persönliche Selbstbewusstsein."

${ }^{20}$ J.G. Fichte, Zurückforderung der Denkfreiheit, p. 11. 
modo, si el ciudadano no está de acuerdo con las normas dictadas por el soberano, puede dar el contrato social por anulado. ${ }^{21}$

Con estos principios en mente, Fichte delineó en estos primeros escritos una teoría del Estado justo. En este marco, los ciudadanos tienen un derecho inalienable a obedecer sólo a su propia ley moral, y también un derecho alienable a la acción externa (äussere Handlung). ${ }^{22}$ El motivo de la publicación de la Zurückforderung der Denkfreiheit fue justamente la defensa de la libertad de pensamiento, considerada por Fichte como inalienable. ${ }^{23}$ La libertad de expresión es indispensable para el desarrollo de la moralidad del individuo, porque si no puede pensar sin restricciones no podrá nunca autolegislarse. Esto exige también la libertad de expresión, porque nadie puede formarse moralmente sin intercambiar su parecer con los demás y actuar con base en las mejores razones de que disponga. ${ }^{24} \mathrm{El}$ contrato social prevé únicamente la cesión de los derechos a la acción externa, pero sólo en el caso en que los demás también lo hagan. ${ }^{25}$ Pero de la defensa de la Revolución Francesa no se sigue una propuesta de democracia directa. Por el contrario, Fichte sostiene que la única forma practicable de gobierno consiste en ceder todo el poder a un ciudadano o a un grupo de ellos. Se trata del príncipe (Fürst), que recibe sus atribuciones por transferencia de la sociedad y no ya por sucesión hereditaria. ${ }^{26}$ Dado que en el Estado fichteano debe gobernar la ley y ya no la voluntad arbitraria de tal o cual individuo particular, y puesto que los nobles han mostrado siempre una voluntad caprichosa y cambiante, se descarta de plano la posibilidad de que accedan a los cargos públicos. ${ }^{27}$

Considero que la teoría del Estado sostenida por el joven Fichte dejó abierto el problema de la estabilidad de una comunidad en la que los ciudadanos no estuvieran dispuestos a autolegislarse en lo moral. Fichte intentó responder a esta cuestión construyendo una teoría del Estado que sea totalmente independiente de la ética, en su Grundlage des Naturrechts de 1796/1797. Para lograr esta meta decidió elaborar su teoría del derecho a partir de la estructura misma del yo, común a

${ }^{21}$ G. Rametta, "Politik der Vernunft und Vernunftstaat bei Fichte (1793-1808)", p. 230.

${ }^{22}$ J.G. Fichte, Zurückforderung der Denkfreiheit, pp. 12-13.

${ }^{23}$ Véase en especial J.G. Fichte, Zurückforderung der Denkfreiheit, p. 15.

${ }^{24}$ Ibid., pp. 13-17, 24.

${ }^{25}$ Ibid., p. 13.

${ }^{26}$ Ibid., pp. 13 y 32 .

${ }^{27}$ J.G. Fichte, Beitrag zur Berichtigung der Urtheile des Publicums über die französische Revolution, p. 243. 
todos los seres racionales, para mostrar que la legalidad es una condición misma de la autoconciencia.

La argumentación podría sintetizarse como sigue. El yo consiste en la autoconciencia, es decir, en la atribución a sí mismo de la capacidad de actuar en el mundo sensible. ${ }^{28}$ Ahora bien, el yo no puede atribuirse a sí mismo esta capacidad antes de ser autoconsciente, lo que implica que sólo podrá saber que tiene esta facultad por medio de otro, en la forma de una relación de reconocimiento. ${ }^{29} \mathrm{El}$ otro debe exhortar de alguna manera al yo para que pueda volverse autoconsciente, porque la relación de exhortación (Aufforderung) lleva implícita en sí misma el reconocimiento del otro como un ser racional y libre, o sea como agente. $^{30}$

Por otro lado, el derecho es la condición de posibilidad última de la relación de mutuo reconocimiento entre las autoconciencias, porque el reconocimiento del otro como un ser racional y, por lo tanto, como un sujeto autodeterminado, implica necesariamente el respeto de su esfera de libertad de acción. Sería contradictorio que un sujeto exhortara al otro a la acción libre y a la vez lo coaccionara para que siguiera tal o cual curso de acción. ${ }^{31}$ Por lo tanto, la relación intersubjetiva de reconocimiento debe estar necesariamente regida por una ley, que Fichte denomina "principio del derecho" (Rechtsatz), según el cual cada individuo debe limitar su esfera de acción teniendo en cuenta la posibilidad de una esfera igual para el otro. ${ }^{32}$

La comunidad que se forma a partir del respeto del principio del derecho es una comunidad legal, que permanece existiendo mientras los sujetos se comporten según esta regla. ${ }^{33}$ De este modo, se ha deducido el derecho natural a la acción libre a partir del concepto de autoconciencia. De esto se desprende una consecuencia importante para la teoría fichteana, que consiste en que el yo no puede existir como tal si no respeta el derecho de los demás a la libertad de acción.

Pero este esquema de relación intersubjetiva, basado en el reconocimiento de la libertad del otro como un fin en sí mismo, cambia radicalmente en la teoría fichteana de la propiedad. Una vez reconocido el derecho del yo a la acción libre, Fichte considera necesario precisar las

${ }^{28}$ J.G. Fichte, Grundlage des Naturrechts, p. 17.

${ }^{29}$ Ibid., p. 33.

${ }^{30}$ Ibid., p. 33. Cfr. A. Honneth, "Die transzendentale Notwendigkeit von Intersubjektivität (Zweiter Lehrsatz, § 3)", pp. 63-80.

${ }^{31}$ J.G. Fichte, Grundlage des Naturrechts, p. 34.

${ }^{32}$ Ibid., p. 52.

${ }^{33}$ Ibid., p. 46.

Diánoia, vol. LVI, no. 66 (Mayo 2011). 
condiciones que deben ser respetadas para que pueda hacerse efectivo este derecho. Ante todo, debe garantizarse una determinada cantidad de propiedad, para que el sujeto pueda realizar efectivamente sus acciones en el mundo sensible. ${ }^{34} \mathrm{Si}$ el yo no sabe que contará en el futuro con tales y cuales bienes, no podrá siquiera ponerse el fin de realizar la acción. Pero esto, a su vez, es posible sólo si se excluye a los demás del uso de estos bienes que el yo necesita para realizar su acción. ${ }^{35}$ Por otro lado, esto es posible únicamente en una organización política del tipo de un Estado, donde haya un tercero que medie en los conflictos por la propiedad que puedan surgir entre los ciudadanos. ${ }^{36}$

El Estado debe garantizar el derecho inalienable de todo ciudadano a la existencia, es decir, a satisfacer el hambre y la sed, así como a prevenir las enfermedades evitables, porque nadie puede proponerse el fin de realizar una acción si no sabe si existirá o no al momento de llevarla a cabo. ${ }^{37}$ De este modo, cada ciudadano debe poder vivir de su trabajo, para que el Estado garantice los derechos que le corresponden a cada uno, según el dictado de la razón. ${ }^{38}$ Como argumenta Fichte, no puede decirse que un sastre tiene derecho a ejercer su oficio si el Estado no le

${ }^{34}$ Ante todo, debe garantizarse su derecho a la existencia (J.G. Fichte, Grundlage
des Naturrechts, p. 212).
${ }^{35}$ Por esta razón Braun caracteriza el contrato de ciudadanía como un "Versicherungsvertrag" (J. Braun, Freiheit, Gleichheit, Eigentum. Grundfragen des Rechts im Lichte der Philosophie J.G. Fichtes, pp. 31-32). Por razones de espacio no puedo exponer exhaustivamente aquí la teoría fichteana de la propiedad, para lo cual remito a J.-Ch. Merle, "Le Droit de proprieté chez Fichte", p. 120; "Eigentumsrecht (§§ 18-19)", pp. 159-161. También es ilustrativa la exposición de H. Verweyen, Recht und Sittlichkeit in J.G. Fichtes Gesellschaftslehre, pp. 101-132.

${ }^{36}$ J.G. Fichte, Grundlage des Naturrechts, p. 157.

${ }^{37}$ Ibid., p. 211. Fichte complementa en otros textos el ideal del mero Lebenkönnen con el más ambicioso de una angenehmes Leben. Fichte toma este ideal del pietismo, esto es, una vida en la cual el hombre no sea tratado como un animal de carga (Lasttier), sino que pueda llevar una vida decente de acuerdo a su función social (p.ej., un campesino puede darse por satisfecho con una alimentación vegetariana y puede ir a trabajar con zapatos de madera y usar en los días festivos zapatos de cuero, mientras que el sabio necesita una alimentación variada y un reine Umgebung). Cada uno debe poder trabajar con alegría y poder ver el cielo en condiciones de tranquilidad (J.G. Fichte, Der geschlossene Handelsstaat, pp. 414 y ss.). Cfr. el ejemplo de los Herrenhuter (Excurse zur Staatslehre, p. 609).

${ }^{38}$ J.G. Fichte, Grundlage des Naturrechts, p. 212; J.G. Fichte, Der geschlossene Handelsstaat, pp. 446-447. Esta concepción del Estado influyó poderosamente en el socialismo, en autores tales como Lasalle, entre otros. Al respecto puede consultarse el interesante trabajo de A. Damiani, "El Fichte de Lasalle: antecedentes e influencias". 
garantiza que su trabajo será demandado y remunerado. ${ }^{39} \mathrm{Al}$ derecho a la existencia le corresponde la obligación de trabajar (Arbeitspflicht) que también tienen todos los habitantes del Estado, a fin de sostener a la comunidad. ${ }^{40}$ El Estado debe dividir y coordinar el trabajo de todos, de modo tal que haga efectivo el principio del derecho y garantice a todos la cantidad de propiedad que les corresponde. Para lograr esta finalidad, Fichte considera necesario que la sociedad se divida en tres grupos: los productores (que obtienen las materias primas, sobre todo a través de la agricultura y la ganadería), los artesanos (quienes elaboran las materias primas para producir diversos objetos e instrumentos) y los comerciantes (a quienes compete el intercambio entre las materias primas de los productores y los objetos de los artesanos). ${ }^{41}$

La idea del Estado que Fichte desarrolla está fundada en el supuesto del egoísmo universal, que exige la unificación de los poderes ejecutivo, legislativo y judicial en la persona del gobernante. La razón de esto radica en que los ciudadanos deben ser controlados continuamente, a fin de evitar que den rienda suelta a su egoísmo e intenten usurpar la libertad y la propiedad de los demás. ${ }^{42} \mathrm{El}$ ejercicio de la soberanía popular queda restringido a los casos excepcionales en que el gobierno no cumpla con el principio constitucional de aplicar la ley con eficiencia, preservando la propiedad de todos y cada uno de los ciudadanos. ${ }^{43} \mathrm{El}$ eforato es el organismo encargado de controlar la constitucionalidad de los actos de gobierno y también de llamar a la asamblea popular para que los ciudadanos mismos emitan un veredicto en caso de tener serias sospechas de irregularidades. ${ }^{44}$ Es decir, el pueblo se disuelve como comunidad cuando delega su poder en el gobernante, pero vuelve a constituirse como tal con ocasión del juicio político al gobierno, para luego retornar a su estado inicial de dispersión. ${ }^{45}$

Sin embargo, Fichte irá cambiando su posición a lo largo de su obra. En Das System der Rechtslehre de 1812, admite que, en el marco de su

${ }^{39}$ J.G. Fichte, Grundlage des Naturrechts, p. 213.

${ }^{40}$ Ibid., p. 214.

${ }^{41}$ Ibid., pp. 217 y ss.; J.G. Fichte, Der geschlossene Handelsstaat, pp. 403-406.

${ }^{42}$ J.G. Fichte, Grundlage des Naturrechts, p. 166.

${ }^{43}$ Por esa razón, F. Oncina Coves dice que el ejercicio de la soberanía popular es el derecho del no derecho ("Fichtes Kritik des aufklärerischen Republikanismus", pp. 224-225).

${ }^{44}$ J.G. Fichte, Grundlage des Naturrechts, pp. 161, 163, 442-443. Aquí se inspira Fichte en el interdicto eclesiástico, que prevé la suspensión de las funciones de un cargo, a fin de asegurarse la obediencia que necesita (Grundlage des Naturrechts, p. 172).

${ }^{45}$ Ibid., p. 177.

Diánoia, vol. LVI, no. 66 (Mayo 2011). 
propuesta, el eforato puede ser coaccionado por el poder ejecutivo para evitar el pronunciamiento del interdicto, porque se trata de un poder meramente negativo. Dicho de otro modo, no existe ninguna garantía de que pueda cumplir con el mandato constitucional de controlar al gobierno, porque el gobierno monopoliza el poder de coacción. ${ }^{46}$ Por otro lado, en el caso de que convocara con éxito a la asamblea popular y ésta estuviera en condiciones de dar su veredicto libremente, su juicio sería correcto desde el punto de vista formal (porque el pueblo es el origen de toda soberanía), pero no necesariamente desde el punto de vista material, es decir, en relación con la determinación de iguales esferas de libertad para los ciudadanos. En este sentido, es mejor el juicio de un grupo de ciudadanos cultos que el de una mera mayoría. ${ }^{47}$

La única garantía de que el gobierno se cuidará de traicionar el mandato popular consiste en que esté bajo la vigilancia de un grupo de ciudadanos con el entendimiento bien formado; esto es, una masa crítica que esté dispuesta a llamar la atención del gobierno en caso de que tuerza el rumbo y de convocar al pueblo si aquél no escucha sus advertencias. Más aún, debería avanzarse en la educación de todo el pueblo, para que el contralor del gobierno sea una realidad y se avance en el respeto de los derechos de ciudadanía. ${ }^{48}$

Por otro lado, en su Staatslehre de 1813, Fichte considera necesario ampliar las funciones del Estado, tal como las definió en el Grundlage des Naturrechts de 1796/1797. Allí se garantizaba la propiedad de todos por medio de la utilización del egoísmo racional de los ciudadanos, coaccionándolos por medio de la ley para que sirvan al bien común. Pero en la Staatslehre de 1813, Fichte argumenta que la protección de las condiciones materiales de la libertad no es suficiente para que el ciudadano pueda gozar de su derecho fundamental, que consiste en obedecer exclusivamente la ley que esté de acuerdo con sus convicciones. ${ }^{49}$

El gobernante o Zwingherr deberá dar razones respecto de sus decisiones a quien se lo pida, por lo cual deberá poseer el entendimiento mejor formado de su tiempo, de acuerdo con el progreso en el que se hallen la ciencia y la técnica en ese momento. Por esta razón, el gobernante deberá ser elegido por aquellos que tengan como oficio evaluar

${ }^{46}$ J.G. Fichte, Das System der Rechtslehre, p. 632.

${ }^{47}$ Ibid., p. 633.

${ }^{48}$ J.G. Fichte, Das System der Rechtslehre, pp. 633-634. Fichte argumenta en este sentido también en Reden an die deutsche Nation, pp. 364-366.

${ }^{49}$ Con palabras de Fichte: "zu gehorchen ihrer eigenen Einsicht" (J.G. Fichte, Die Staatslehre, p. 437). 
el nivel de desarrollo del entendimiento de los ciudadanos; esto es, por los maestros (Lehrer).$^{50}$ Finalmente, en la medida en que el pueblo sea educado de modo tal que comprenda que la ley que se le impone es justa, la coacción misma será cada vez menos necesaria, hasta desaparecer completamente luego del proceso histórico del establecimiento de un Estado racional. ${ }^{51}$

La finalidad del Estado consiste, entonces, en promover el desarrollo de la especie humana hasta que llegue a ser la expresión misma de la razón en la historia, en la medida en que logre que los ciudadanos organicen sus relaciones con libertad y de acuerdo con la razón. ${ }^{52}$ Por este motivo, la promoción de la cultura es una finalidad insoslayable del Estado, para lo cual deberá cuidar de la subsistencia y la autoconservación de los ciudadanos. ${ }^{53}$ Pero la educación no puede planificarse de cualquier manera, sino que debe apuntar a que el ciudadano pueda utilizar su razón de modo autónomo y libre, ingresando por sí mismo en el mundo del pensamiento y la moralidad. ${ }^{54}$

Fichte propone un camino pedagógico para la construcción de un Estado racional o absoluto, cuyo mediador privilegiado es la nación alemana, porque el lenguaje de los germanos es el único que permite el acceso al mundo de la cultura, el pensamiento y la moralidad. ${ }^{55}$ La idea fichteana de la nacionalidad no tiene una base étnica, sino más bien lingüística, porque el lenguaje es lo que permite a los hablantes compartir una misma concepción del mundo. ${ }^{56}$ El lenguaje está estructurado de modo tal que la manera en que se designan los objetos en la experiencia sensible es determinante para la denominación de los conceptos y las ideas del mundo suprasensible del pensamiento y la moralidad, porque estos últimos se piensan por analogía con aquellos. ${ }^{57}$

Los alemanes han incorporado a su lenguaje aquella experiencia de la primera comunidad política que construyeron, consistente en una federación de Estados gobernados cada uno por un caudillo con pode-

${ }^{50}$ Ibid., SW, IV, 449; Excurse zur Staatslehre, p. 579.

${ }^{51}$ Ibid., p. 574.

52 J.G. Fichte, Die Grundzüge des gegenwärtigen Zeitalters, p. 144.

${ }^{53}$ Ibid., pp. 145-147.

${ }^{54}$ J.G. Fichte, Die Staatslehre, p. 591. En este punto, Fichte se declara deudor de Pestalozzi (J.G. Fichte, Reden an die deutsche Nation, pp. 403-404), cuya propuesta intentará ampliar desde los destinatarios primigenios (los niños pobres y abandonados) hasta la educación de todos los ciudadanos alemanes.

${ }^{55}$ J.G. Fichte, Reden an die deutsche Nation, pp. 324-325.

${ }^{56}$ Ibid., pp. 313-314.

${ }^{57}$ Ibid., pp. 315-318.

Diánoia, vol. LVI, no. 66 (Mayo 2011). 
res limitados. ${ }^{58}$ En esta organización primitiva cada miembro se sentía identificado con el destino de todos, y experimentaba la necesidad y el dolor de los demás como si fueran propios. ${ }^{59}$ Esta experiencia originaria permitió al lenguaje alemán el acceso al mundo suprasensible y la construcción de una moralidad fundada en una comunidad de legisladores racionales que se tratan mutuamente como iguales. La Reforma luterana fue un producto histórico de esta concepción, que trajo como consecuencia la exigencia de la formación moral de todo el pueblo y de su participación en la interpretación misma de las Escrituras. ${ }^{60}$

Por el contrario, los pueblos que hablan lenguas romances no han tenido la posibilidad de acceder al pensamiento de lo suprasensible y no han podido ser una manifestación de la razón en la historia. La causa de esto es que estas lenguas derivan del latín, una lengua muerta que impide a los hablantes el contacto con la experiencia sensible originaria que hizo posible la designación de los objetos y las ideas del mundo suprasensible. ${ }^{61}$ De todo lo dicho hasta aquí Fichte deduce que sólo la nación alemana podrá llevar a la humanidad hacia su desarrollo pleno. ${ }^{62} \mathrm{Al}$ Estado le corresponde garantizar las condiciones para que la nación alemana pueda desarrollarse; es decir, debe cuidar la autoconservación de los ciudadanos, así como la educación de todos en establecimientos públicos donde se los forme en los valores de la germanidad y en la pureza del lenguaje nacional. ${ }^{63}$

\section{Consideraciones finales}

Luego de comparar la concepción del Estado que sostiene Fichte en sus escritos de 1793 y 1794 con la expuesta en la Ethik des reinen Willens, puede concluirse que ambas presentan una semejanza notable. En los dos casos, la autolegislación moral de los ciudadanos desempeña un papel fundamental. Sin embargo, la relación entre la autolegislación de los ciudadanos y el Estado es diferente en cada teoría. En la Ethik des reinen Willens la moralidad constituye el ámbito mismo en el que se desarrolla la autoconciencia, por medio de la participación de todos los ciudadanos en la formación y expresión de la voluntad estatal, es decir, en la tarea de la legislación. De este modo, Cohen considera

\footnotetext{
${ }^{58}$ Ibid., p. 313.

${ }^{59}$ Ibid., pp. 272-273.

${ }^{60}$ Ibid., p. 355.

${ }^{61}$ Ibid., pp. 336-337.

${ }^{62}$ Ibid., pp. 341-342.

${ }^{63}$ Ibid., pp. 422-427.
} 
que el Estado y el sistema legal son producto de la autolegislación de los ciudadanos y, por ello, son el lugar donde pueden ejercer su autonomía moral.

También la idea fichteana del Estado de 1793/1794 se funda en la necesidad de proteger la autolegislación moral de los ciudadanos. En los textos de este periodo, Fichte sostiene que el Estado debe garantizar las condiciones para el desarrollo de la moralidad en los ciudadanos, entre ellas la libertad de expresión y ciertos derechos de propiedad. Pero, a diferencia de la Ethik des reinen Willens de Cohen, Fichte no considera que la vida estatal sea el medio en el que se realiza la autolegislación moral de los ciudadanos, porque el Estado es el producto de un contrato en el que sólo se ceden los derechos alienables, es decir las condiciones externas de la acción, pero no aquellas que pertenecen al fuero interno (por ejemplo, la libertad de conciencia), que es el ámbito propio de la moralidad. Por lo tanto, en el primer Fichte el Estado permanece como una esfera externa a la moral.

En el Grundlage des Naturrechts de 1796/1797, Fichte desarrolla una teoría aún más compleja del Estado. A partir de lo desarrollado en la sección anterior de este trabajo estamos en condiciones de afirmar que existen diferencias importantes entre las concepciones del Estado defendidas por Cohen y por la teoría fichteana del derecho natural de 1796/1797. La idea del Estado se deriva en Cohen de su teoría de la relación legal en general y de la persona jurídica en particular. Por otro lado, la teoría fichteana de un gobierno que unifique los tres poderes es una exigencia del supuesto motivacional del egoísmo universal, con la reducción consiguiente del ejercicio de la soberanía popular sólo a casos excepcionales.

Cohen entiende la relación legal como la integración de sus miembros en una unidad superior, que es la voluntad declarada y acordada por todos. Se trata del producto de la deliberación de todos y es una persona jurídica, un sujeto legal con carácter ficticio. La persona jurídica representa los intereses de todos sus miembros, por eso Cohen la opone a la figura legal de la persona física, que hace referencia a los intereses del individuo particular, tomado aisladamente de su relación con los demás.

La persona jurídica necesita entonces la participación de sus integrantes en la elaboración de sus reglas, en calidad de colegisladores. La persona jurídica de la cooperativa es el modelo que sirve a Cohen de inspiración para construir su teoría del Estado. Esto no debe sorprendernos, dado que la persona jurídica tiene la estructura de toda relación legal y el Estado es el conjunto de todas las relaciones legales, así como 
de las condiciones de su producción. Por lo tanto, el Estado se funda sobre la actividad de colegislación de los ciudadanos en el Parlamento y en las diversas instituciones que gobiernan la vida social (entre ellas, las cooperativas).

La teoría expuesta en el Grundlage des Naturrechts es diferente, porque Fichte ha deducido la necesidad de una relación legal que garantice la relación de mutuo reconocimiento, que a su vez es la condición de posibilidad de la autoconciencia del yo. Dicho de otro modo, Fichte arguye que es incoherente que un yo reconozca al otro como un ser libre y a la vez le quite su esfera de acciones posibles. La razón de esto es que el reconocimiento es una práctica social y presupone cierta coherencia entre la idea que se quiere comunicar por medio de la acción y la forma de la acción misma. Esto significa que la deducción de la relación culmina con el reconocimiento de la libertad del otro como un fin en sí mismo.

Pero luego Fichte advierte que no es posible garantizar la esfera de acciones libres del otro si no es en una organización política que realice la voluntad común de hacer respetar el derecho. La razón de esto es que la comunidad legal no queda garantizada por la mera sumisión voluntaria de sus miembros al principio del derecho y a la ley de la coherencia consigo mismo. De allí la necesidad de introducir el derecho de coacción, para la eventualidad de que alguno de los miembros abandone su compromiso de cumplir con el principio del derecho. Pero el derecho de coacción presupone el egoísmo universal, porque está orientado a utilizar el autointerés de cada ciudadano para determinarlo en orden a la voluntad común. De este modo, cada ciudadano reconocerá la libertad del otro no ya como un fin en sí mismo, sino más bien como un medio para proteger la propia esfera de acción. No obstante, no puede confiarse en que estos ciudadanos egoístas vayan a elaborar y aplicar la ley con imparcialidad; por eso es necesario que deleguen toda su libertad en un tercero que unifique los poderes ejecutivo, legislativo y judicial en sí mismo. Al final de la investigación de Fichte desaparece aquella relación dialógica de la comunidad legal y queda sólo una multitud disgregada y sometida al arbitrio de un gobierno integrado por unos pocos.

Fichte está lejos de la idea que tiene Cohen de la ciudadanía como autolegislación, y del Estado como la tarea de la conformación de una voluntad común expresa en la actividad del Parlamento. La razón de esto reside en que Fichte no confiere autonomía al poder legislativo y, por lo tanto, no puede construir un espacio público de debate y de toma de decisiones, porque considera que los ciudadanos no pueden legislar 
de acuerdo con la Constitución. Fichte supone que, si se deja a los ciudadanos a su libre arbitrio, entonces perseguirán implacablemente su autointerés y sabotearán la estabilidad de la comunidad legal, poniendo en peligro la propiedad y la libertad de todos los demás. Por lo tanto, la exigencia de imparcialidad en la legislación y la administración de justicia requiere que estas funciones sean la prerrogativa de un tercero que no esté vinculado estrechamente con la vida del pueblo.

Los últimos escritos de Fichte sobre el derecho y el Estado también presentan diferencias de fondo con la Ethik des reinen Willens. Mientras que Cohen identifica el Estado con la realización de la moralidad misma, Fichte considera que el Estado tiene la función de proveer las condiciones para el desarrollo de la moralidad en la historia, de modo tal que la coacción se vuelva cada vez más superflua. En la teoría fichteana el Estado no es ya un fin en sí mismo, como ocurre en la de Cohen, sino que está subordinado a la finalidad suprema de convertirse en la manifestación de la razón misma en la historia. Fichte sostiene que el Estado está llamado a promover el desarrollo pleno de las potencialidades del género humano, inscritas en su naturaleza. El Estado ideado por Cohen es la mediación única de la universalidad moral; por el contrario, el Estado de Fichte es un garante de la autoconservación y la educación de los ciudadanos, pero no puede nunca ser identificado con la moralidad.

\section{BIBLIOGRAFÍA}

Fuentes

Cohen, Hermann, Einleitung mit kritischem Nachtrag, Verlag von Friedrich Brandstetter, Leipzig, 1914.

_ Ethik des reinen Willens, Bruno Cassirer, Berlín, 1907; reimpreso en Herman Cohen. Werke, Georg Olms Verlag, Hildesheim-Zurich-Nueva York, 2002, t. 7.

_- Logik der reinen Erkenntnis, Bruno Cassirer, Berlín, 1914; reimpreso en Herman Cohen. Werke, Georg Olms Verlag, Hildesheim-Zurich-Nueva York, 2005, t. 6.

Fichte, Johann Gottlieb, Beitrag zur Berichtigung der Urtheile des Publicums über die französische Revolution, 1794, en Sämmtliche Werke, ed. Immanuel Hermann Fichte, Walter de Gruyter, Berlín, 1971, t. VI.

_ _ Das System der Rechtslehre (1812), en Sämmtliche Werke, ed. Immanuel Hermann Fichte, Walter de Gruyter, Berlín, 1971, t. X.

— Der geschlossene Handelsstaat (1800), en Sämmtliche Werke, ed. Immanuel Hermann Fichte, Walter de Gruyter, Berlín, 1971, t. III.

Diánoia, vol. LVI, no. 66 (Mayo 2011). 
Fichte, Johann Gottlieb, Die Grundzüge des gegenwärtigen Zeitalters (1806), en Sämmtliche Werke, ed. Immanuel Hermann Fichte, Walter de Gruyter, Berlín, 1971, t. VII.

— Die Staatslehre, oder über das Verhältniss des Urstaates zum Vernunftreiche, in Vorlesungen, gehalten im Sommer 1813 auf der Universität zu Berlin (1813), en Sämmtliche Werke, ed. Immanuel Hermann Fichte, Walter de Gruyter, Berlín, 1971, t. IV.

_- Excurse zur Staatslehre, en Sämmtliche Werke, ed. Immanuel Hermann Fichte, Walter de Gruyter, Berlín, 1971, t. VII.

_- Grundlage des Naturrechts nach der Prinzipien der Wissenschaftslehre (1796/1797), en Sämmtliche Werke, ed. Immanuel Hermann Fichte, Walter de Gruyter, Berlín, 1971, t. III.

— Reden an die deutsche Nation (1808), en Sämmtliche Werke, ed. Immanuel Hermann Fichte, Walter de Gruyter, Berlín, 1971, t. VII.

— Zurückforderung der Denkfreiheit von den Fürsten Europens, die sie bisher unterdrückten (1793), en Sämmtliche Werke, ed. Immanuel Hermann Fichte, Walter de Gruyter, Berlín, 1971, t. VI.

Bibliografía secundaria

Adelmann, Dieter, "Einheit des Bewusstseins als Grundproblem der Philosophie Hermann Cohens", tesis doctoral, Heidelberg, 1968.

Batscha, Zwi, Gesellschaft und Staat in der politischen Philosophie Fichtes, Europäische Verlagsanstalt, Fráncfort del Meno, 1970.

Brandt, Reinhard y Franz Orlik (comps.), Philosophisches Denken - Politisches Wirken. Hermann Cohens Kolloquium Marburg 1992, Georg Olms Verlag, Hildesheim-Zurich-Nueva York, 1993.

Braun, Johann, Freiheit, Gleichheit, Eigentum. Grundfragen des Rechts im Lichte der Philosophie J.G. Fichtes, J.C.B. Mohr (Paul Siebeck), Tubinga, 1991.

Damiani, Alberto Mario, "El Fichte de Lasalle: antecedentes e influencias", Deus Mortalis, no. 7, 2008, pp. 253-275.

De Pascale, Carla, Erich Fuchs, Marco Ivaldo y Günther Zöller (comps.), Fichte und die Aufklärung, Georg Olms, Hildesheim/Zurich/Nueva York, 2004.

Dreyer, Metchild, Die Idee Gottes im Werk Hermann Cohens, Verlag Anton Hain Meisenheim GmbH, Königstein/Ts., 1985.

Fiorato, Pierfrancesco, Geschichtliche Ewigkeit. Ursprung und Zeitlichkeit in der Philosophie Hermann Cohens, Königshausen y Neumann, Würzburg, 1993.

Fonnesu, Luca, "Die Aufhebung des Staates bei Fichte", Fichte-Studien, vol. 11, 1997, pp. 85-97.

Gadamer, Hans-Georg, "Subjektivität und Intersubjektivität, Subjekt und Person", en Hans Georg Gadamer: Gesammelte Werke, t. 10, Hermeneutik im Rückblick, J.C.B. Mohr (Paul Siebeck), Tubinga, 1995, pp. 87-99.

Giesecke, Peter, "Kant und der Sozialismus. Studien zum Marburger Neukantianismus, Philosophisches Kritizismus und Kritischen Rationalismus", tesis doctoral, Munich, 1990. 
Görland, Albert, "Hermann Cohens systematische Arbeit im Dienste des kritischen Idealismus", Kant-Studien, vol. 17, 1912, pp. 222-251.

Heidegger, Martin, "Phänomenologie und transzendentale Wertphilosophie. Vorlesung Sommersemester 1919", en Gesamtausgabe, vols. 56/57, Zur Bestimmung der Philosophie, Vittorio Klostermann, Fráncfort del Meno, 1987, pp. 119-203.

Holzhey, Helmut, Cohen und Natorp, Schwabe, Basilea-Stuttgart, 1986, 2 vols.

— - "Hermann Cohen: der Philosoph in Auseinandersetzung mit dem politischen und gesellschaftlichen Probleme seiner Zeit", en Brandt y Orlik (comps.), Philosophisches Denken - Politisches Wirken. Hermann Cohens Kolloquium Marburg 1992, pp. 15-36.

- (comp.), Auslegungen. Band 4. Hermann Cohen, Peter Lang, Fráncfort del Meno-Berlín-Berna-Nueva York-París-Viena, 1994.

Honneth, Axel, "Die transzendentale Notwendigkeit von Intersubjektivität (Zweiter Lehrsatz, § 3)", en Jean-Christophe Merle (comp.), Johann Gottlieb Fichte. Grundlage des Naturrechts, Akademie Verlag, Berlín, 2001, pp. 63-80 (Klassiker Auslegen, t. 24).

Lasson, Adolf, Johann Gottlieb Fichte im Verhältnis zu Kirche und Staat, Scientia Verlag Aalen, Darmstadt, 1968; reimpresión de la edición de Berlín, 1863.

Lisser, Kurt, Der Begriff des Rechts bei Kant. Mit einem Anhang über Cohen und Görland, Verlag von Reuter und Reichard, Berlín, 1922.

Lübbe, Hermann, "Die politische Theorie des Neukantianismus und des Marxismus", en Holzhey (comp.), Auslegungen. Band 4. Hermann Cohen, pp. $229-246$.

—_, "Neukantianische Sozialismus", en Hans Ludwig Ollig (comp.), Materialien zur Neukantianismus Diskussion, Wissenschaftliche Buchgesellschaft, Darmstadt, 1987, pp. 219-263.

Maesschalk, Marc, Droit et creation sociale chez Fichte. Une philosophie moderne de l'action politique, Éditions de L'Institut Supérieur de Philosophie LouvainLa-Neuve/Éditions Peeters, Lovaina-París, 1996.

Merle, Jean-Christoph, "Eigentumsrecht (§§ 18-19)", en Jean-Christoph Merle (comp.), Johann Gottlieb Fichte. Grundlage des Naturrechts, Akademie Verlag, Berlín, 2001, pp. 159-172 (Klassiker Auslegen, 24).

_-, "Le Droit de proprieté chez Fichte", en Myriam Bienenstock y Michèle Crampe-Casnabet, Dans quelle mesure la philosophie est pratique. Fichte, Hegel, con la colaboración de Jean-François Goubet, Ens Éditions, Fontenay/ Saint-Cloud, 2000, pp. 159-172.

Meyer, Thomas, "Eine unzeitgemäße Intervention, die an der Zeit war. Hermann Cohens neukantianischer Sozialismus und die sozialdemokratische Ideologie im Kaiserreich", en Brandt y Orlik (comps.), Philosophisches Denken - Politisches Wirken. Hermann Cohens Kolloquium Marburg 1992, pp. 257-269.

Müller, Claudius, Die Rechtsphilosophie des Marburger Neukantianismus. Naturrecht und Rechtpositivismus in der Auseinandersetzung zwischen Hermann Co-

Diánoia, vol. LVI, no. 66 (Mayo 2011). 
hen, Rudolf Stammler und Paul Natorp, J.C.B. Mohr (Paul Siebeck), Tubinga, 1994.

Natorp, Paul, "Hermann Cohens philosophische Leistung unter dem Gesichtspunkte des Systems", Philosophische Vorträge der Kant-Gesellschaft, Berlín, 1918, no. 21; reimpreso en Holzhey (comp.), Auslegungen. Band 4. Hermann Cohen, Peter Lang, Fráncfort del Meno/Berlín/Berna/Nueva York/París/Viena, 1994, pp. 75-103.

Ollig, Hans Ludwig, Der Neukantianismus, Metzler, Stuttgart, 1979.

— - Religion und Freiheitsglaube, Forum Academicum in der Verlagsgruppe Athenäum, Hain, Scriptor, Hanstein, 1979.

Oncina Coves, Faustino, "Fichtes Kritik des aufklärerischen Republikanismus", en De Pascale, Fuchs, Ivaldo y Zöller (comps.), Fichte und die Aufklärung, pp. 213-226.

- "Wahlverwandtschaften zwischen Fichtes, Maimons und Erhardts Rechtslehren", Fichte-Studien, vol. 11, 1997, pp. 63-84.

Pascher, Manfred, Hermann Cohens Ethik als Gegenentwurf zur Rechtsphilosophie Hegels, Verlag des Instituts für Sprachwissenschaft der Universität Innsbruck, Innsbruck, 1992.

Poma, Andrea, The Critical Philosophy of Hermann Cohen, State University of New York Press, Albany, 1997.

Rametta, Gaetano, "Politik der Vernunft und Vernunftstaat bei Fichte (17931808)", en De Pascale, Fuchs, Ivaldo y Zöller (comps.), Fichte und die Aufklärung, pp. 227-241.

Schmid, Peter, Ethik als Hermeneutik. Systematische Untersuchungen zu Hermann Cohens Rechts und Tugendlehre, Könighausen und Neumann, Würzburg, 1993.

Schwarzschild, Steven, "The Democratic Socialism of Hermann Cohen", en Holzhey (comp.), Auslegungen. Band 4. Hermann Cohen, pp. 205-227.

Stolzenberg, Jürgen, "Fichte im Neukantianismus. Probleme der Fichte-Rezeption bei Wilhelm Windelband, Heinrich Rickert, Hermann Cohen und Paul Natorp", en Robert Alexy, Lukas Meyer, Stanley Paulson y Gerhard Sprenger (comps.), Neukantianismus und Rechtsphilosophie. Mit einer Einleitung von Stanley Paulson, Nomos Verlagsgesellschaft, Baden-Baden, 2002, pp. 421434.

— - Ursprung und System. Probleme der Begründung systematischer Philosophie im Werk Hermann Cohens, Paul Natorps und beim frühen Martin Heidegger, Vandenhoeck und Ruprecht, Gotinga, 1995.

Vincenti, Luc, Pratique et réalité dans les philosophies de Kant et de Fichte, Éditions Kimé, París, 1997.

Verweyen, Hansjürgen, Recht und Sittlichkeit in J.G. Fichtes Gesellschaftslehre, Karl Alber, Friburgo/Munich, 1975.

Wiedebach, Hartwig, Die Bedeutung der Nationalität für Hermann Cohen, Georg Olms, Hildesheim/Zurich/Nueva York, 1997. 
Winter, Eggert, Ethik und Rechtswissenschaft. Eine historisch-systematische Untersuchung zur Ethik-Konzeption des Marburger Neukantianismus im Werke Hermann Cohens, Duncker und Humblot, Berlín, 1980.

Zaczyk, Rainer, Das Strafrecht in der Rechtslehre J.G. Fichtes, Duncker und Humblot, Berlín, 1981.

Recibido el 30 octubre de 2008; aceptado el 10 de junio de 2010.

Diánoia, vol. LVI, no. 66 (Mayo 2011). 